\title{
羟基磷灰石/氧化铁复合涂层的制备 及其诱导骨生长的生物活性
}

\author{
李龙珠 黄紫洋* 林 辇 曹 媚 林剑群 \\ (福建师范大学化学与化工学院＼cjkstart福州 350007)
}

\begin{abstract}
摘 要 用电泳沉积法制得羟基磷灰石/壳聚糖/氧化铁 $\left(\mathrm{HA} / \mathrm{CS} / \mathrm{Fe}_{2} \mathrm{O}_{3}\right)$ 复合涂层,经 $700{ }^{\circ} \mathrm{C}$ 烧结处理得到 $\mathrm{HA} / \mathrm{Fe}_{2} \mathrm{O}_{3}$ 复合涂层。通过 SEM、EDS、XRD、FT-IR、电化学和万能材料试验机等对复合涂层的表面形貌、物相 组成、抗腐蚀性和结合强度进行了表征和测试,最后采用 $1.5 \mathrm{SBF}$ 浸泡法对复合涂层的生物活性进行了评价。 结果表明,当悬浮液中的 $\mathrm{HA} 、 \mathrm{CS}$ 与 $\mathrm{Fe}_{2} \mathrm{O}_{3}$ 质量比为 100:100: 1 时,所制得的 $\mathrm{HA} / \mathrm{Fe}_{2} \mathrm{O}_{3}$ 复合涂层表面粗䊅, 抗 腐蚀性强,具有良好的诱导骨生长生物活性,基体与复合涂层结合强度可达 $27.5 \mathrm{MPa}$ 。
\end{abstract}

关键词 羟基磷灰石/氧化铁复合涂层,电泳沉积,生物活性

中图分类号:0647.1; TQ174.1

文献标识码:A

文章编号:1000-0518(2013) 10-1149-07

DOI:10. 3724/SP. J. 1095. 2013. 20559

目前已成功应用于临床的医用金属材料主要有金属钛及钛合金, 在其表面上涂覆具有优良生物活 性的差基磷灰石 (HA) 或其它磷酸盐涂层是目前研究较为广泛的课题 ${ }^{[1]}$ 。研究表明 ${ }^{[2]}$, HA 涂层与人体 体液接触后表面逐渐溶解, 诱导形成一层类骨磷灰石, 这一现象与人体骨组织功能相似。新骨可沿 HA 涂层表面生长, 具有骨传导性; 且 HA 涂层具有良好的桥接作用, 当它与骨组织间的间隙为 $2 \mathrm{~mm}$ 时仍然 能够激发骨的生长 ${ }^{[3]}$ 。

成骨微量元素 (osteogenic trace elements, OTE) 是指骨矿物质的磷灰石晶体组成中除了 Ca、P、O 和 $\mathrm{H}$ 元素外的其它 9 种元素 ${ }^{[4]}$,其中 $\mathrm{Zn} 、 \mathrm{Si} 、 \mathrm{Fe}$ 和 $\mathrm{Sr}$ 这 4 种微量元素所占的比例均在 $3 \times 10^{-5}$ 以上, 因而 模拟天然骨磷灰石的成分和结构, 将骨矿物质中的微量元素以化合物形式加人到人工制备的 HA 复合 涂层中, 将可能比纯 HA 涂层更能提高 HA 生物陶瓷材料的生物活性和骨传导性 ${ }^{[5]}$, 由于纯 HA 涂层存 在与基体结合强度低、脆性大和生物活性不高等缺点 ${ }^{[6]}$, 而加人 $\mathrm{Sr}$ 制成的锶磷灰石则具有良好的生物 相容性 ${ }^{[7]}$ 。 $\mathrm{Fe}_{2} \mathrm{O}_{3}$ 在制备微胶囊方面表现出良好的生物相容性 ${ }^{[8]}$, 在 $\mathrm{CaO}-\mathrm{SiO}_{2}-\mathrm{P}_{2} \mathrm{O}_{5}-\mathrm{MgO}-\mathrm{Fe}_{2} \mathrm{O}_{3}$ 体系中 合成的磁性生物活性玻璃陶瓷也具有良好的生物活性 ${ }^{[9]}$, 但对于电泳沉积制得 $\mathrm{HA} / \mathrm{Fe}_{2} \mathrm{O}_{3}$ 复合涂层用 于诱导骨生长方面的研究目前尚未见报道。

本文以纯钛片作为基体, 在其表面电泳沉积过程中加人 $\mathrm{Fe}_{2} \mathrm{O}_{3}$ 形成共沉积复合涂层 $\mathrm{HA} / \mathrm{CS} / \mathrm{Fe}_{2} \mathrm{O}_{3}$, 经后续烧结处理制得表面粗粘、孔洞分布均匀的 $\mathrm{HA} / \mathrm{Fe}_{2} \mathrm{O}_{3}$ 复合涂层。这种结构既有利于骨组织的长 人, 又不明显降低涂层与基体的结合强度。采用 $1.5 \mathrm{SBF}$ 浸泡法评价了成骨微量铁元素化合物对复合涂 层生物活性的影响。

\section{1 实验部分}

\section{1 试剂和仪器}

$\mathrm{HA}$ 粉体 (自制 ${ }^{[10]}$ ), 粒径为 $1 \sim 100 \mathrm{~nm} ; \mathrm{Fe}_{2} \mathrm{O}_{3}$, 分析纯, 粒径 $<38 \mu \mathrm{m}$; 壳聚糖, 分析纯, 脱乙酰度 $96 \%$, 粒径 $<38 \mu \mathrm{m}$; 丙酮、氢氟酸、硝酸、三乙醇胺、正丁醇、盐酸等均为分析纯试剂, 以上试剂均购自中 国医药集团,水溶液均用二次蒸馏水配制。 
FA2004 型电子天平 (上海海康电子仪器厂) ; DDY-6B 型稳压稳流电泳仪 (北京市六一仪器厂) ; Nicolet Aratar 360 型傅里叶红外光谱仪 (美国 Nicolet 公司);JSM-7500F 型冷场发射扫描电子显微镜 (日 本 JEOL 公司); X-Perd MPD 型 X 射线粉末衍射仪 (荷兰 Phillip 公司) ; LR5K PLUS 型万能材料试验机 (英国 Lloyd 材料公司), 按 ASTM F1044-87 标准进行测试;CHI 660C 型电化学工作站(上海辰华)。

\section{$1.2 \mathrm{HA} / \mathrm{Fe}_{2} \mathrm{O}_{3}$ 复合涂层的制备}

1.2.1 电泳沉积制备 $\mathrm{HA} / \mathrm{CS} / \mathrm{Fe}_{2} \mathrm{O}_{3}$ 复合涂层 按文献 $[11]$ 方法处理钛片, 电泳沉积前, 用聚四氟乙烯 生料带缠绕包覆钛片, 控制沉积面积为 $10 \mathrm{~mm} \times 10 \mathrm{~mm}$ 。以三乙醇胺为添加剂, 正丁醇为分散剂, 将 $0.3980 \mathrm{~g} \mathrm{HA}$ 粉体、0.3980 g CS 微粒及 $0.0040 \mathrm{~g} \mathrm{Fe}_{2} \mathrm{O}_{3}$ 粉末加人盛有 $40 \mathrm{~mL}$ 正丁醇的烧杯中, 同时加人 $0.5 \mathrm{~mL}$ 三乙醇胺,将该悬浮液在常温下超声分散 $1 \mathrm{~h}$,密封静置陈化 $24 \mathrm{~h}$ 以上。

以经过预处理的纯钛片为负极, 大面积铂片为正极, 电泳沉积时电极间距 $7.0 \mathrm{~mm}$, 电压 $28 \mathrm{~V}$, 沉积 时间 $60 \mathrm{~s}$, 电泳沉积结束后取出, 用正丁醇蘸洗 1 次, 自然晾干,存放于干燥器中。

1.2 .2 复合涂层的热处理 将电泳沉积制得的 $\mathrm{HA} / \mathrm{CS} / \mathrm{Fe}_{2} \mathrm{O}_{3}$ 复合涂层置于马福炉中通过程序升温进 行烧结处理 ${ }^{[7]}$ 。自然冷却至室温后取出, 保存于干燥器中待用。

1.2.3 $\mathrm{HA} / \mathrm{Fe}_{2} \mathrm{O}_{3}$ 复合涂层的生物活性评价 $1.5 \mathrm{SBF}$ 配制方法如文献 [12] 所述。将经 $700{ }^{\circ} \mathrm{C}$ 烧结处 理的 $\mathrm{HA} / \mathrm{Fe}_{2} \mathrm{O}_{3}$ 复合涂层浸泡在盛有 $50 \mathrm{~mL} 1.5 \mathrm{SBF}$ 的烧杯中, 于电热恒温水浴锅中 (控制水温 $\left.(37 \pm 0.1){ }^{\circ} \mathrm{C}\right)$ 浸泡,每 $2 \mathrm{~d}$ 更换 1 次 $1.5 \mathrm{SBF}$, 浸泡至所需时间后取出试样, 用二次蒸馏水清洗表面, 自 然晾干, 置于干燥器中待用。

\section{2 结果与讨论}

\section{1 复合涂层的 FT-IR 表征}

复合涂层经 $700{ }^{\circ} \mathrm{C}$ 烧结处理 $2 \mathrm{~h}$ 前后的 FT-IR 谱图如图 1 所示。从图 1 可以看出,在烧结处理前复 合涂层中, 2925 及 $2853 \mathrm{~cm}^{-1}$ 处出现的吸收峰为亚甲基的 $\mathrm{C}-\mathrm{H}$ 伸缩振动吸收峰, 在 $1260 \mathrm{~cm}^{-1}$ 处出现 仲醇的 $\mathrm{O}-\mathrm{H}$ 伸缩振动吸收峰, 在 $1631 \mathrm{~cm}^{-1}$ 处出现氨基的 $\mathrm{N}-\mathrm{H}$ 弯曲振动吸收峰, 这些吸收峰为壳聚 糖的特征吸收峰 ${ }^{[11]}$, 表明在电泳沉积过程中壳聚糖也同时沉积到复合涂层中。经 $700{ }^{\circ} \mathrm{C}$ 保温 $2 \mathrm{~h}$ 烧结 处理后 (曲线 $b$ ), 上述 3 处壳聚糖的特征吸收峰均已消失, 壳聚糖的热分解温度为 $260 \sim 350{ }^{\circ} \mathrm{C}^{[10]}$, 说 明涂层中的壳聚糖已在烧结过程中完全分解或燃烧。在 3571 和 $604 \mathrm{~cm}^{-1}$ 处分别出现 $\mathrm{O}-\mathrm{H}$ 的伸缩振 动和弯曲振动吸收峰, 1096、1046、984 和 $569 \mathrm{~cm}^{-1}$ 分别为 $\mathrm{PO}_{4}^{3-}$ 的 $\nu_{4} 、 \nu_{5} 、 \nu_{1}$ 和 $\nu_{3}$ 振动吸收峰, 可见复合涂 层在烧结前后主要成份均包含羟基磷灰石 ${ }^{[13]}$ 。

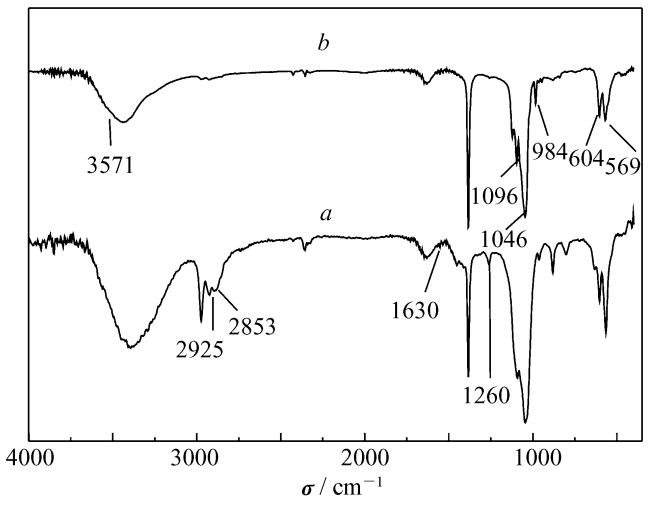

图 1 复合涂层经 $700{ }^{\circ} \mathrm{C}$ 烧结处理 $2 \mathrm{~h}$ 前 $(a)$ 、后 (b) 的 FT-IR 谱图

Fig. 1 FT-IR spectra of composite coatings before $(a)$ and $\operatorname{after}(b)$ sintering at $700{ }^{\circ} \mathrm{C}$ for $2 \mathrm{~h}$

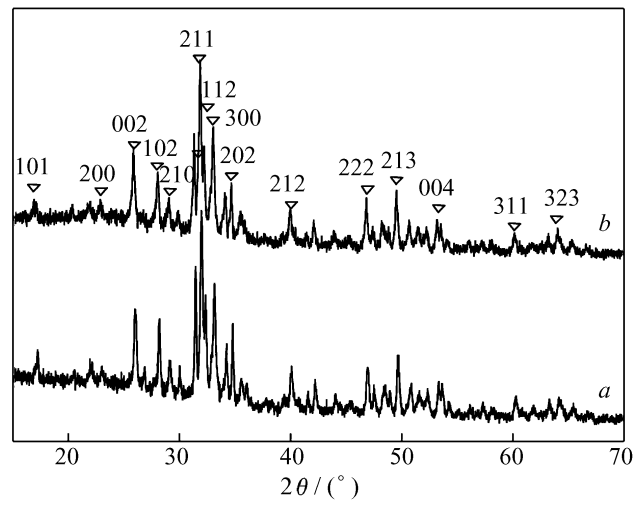

图 2 复合涂层烧结前 $(a)$ 、后 $(b)$ 的 XRD 谱图

Fig. 2 XRD patterns of composite coatings before $(a)$ and $\operatorname{after}(b)$ sintering

\section{2 复合涂层物相及化学组成分析}

复合涂层在烧结前后的 XRD 谱图如图 2 所示。从图 2 可以看出, 烧结前后复合涂层在 $2 \theta$ 为 
$16.8^{\circ} 、 25.9^{\circ} 、 28.1^{\circ} 、 28.9^{\circ} 、 31.8^{\circ} 、 32.2^{\circ} 、 32.9^{\circ} 、 34.1^{\circ} 、 39.2^{\circ} 、 46.7^{\circ} 、 49.5^{\circ} 、 53.2^{\circ} 、 60.4^{\circ}$ 和 $64.2^{\circ}$ 处均出 现六方晶系 HA 的衍射峰, 与标准卡片 (JCPDS 09-0432) 完全吻合, 说明复合涂层中的 HA 的晶型在 $700{ }^{\circ} \mathrm{C}$ 烧结处理 $2 \mathrm{~h}$ 后未发生明显转变。

由 EDS 分析得到的烧结前后复合涂层表面各元素的含量如表 1 所示。从表 1 可以看到,复合涂层 在烧结前后均出现 $\mathrm{O} 、 \mathrm{Ca} 、 \mathrm{P} 、 \mathrm{Ti}$ 和 $\mathrm{Fe}$ 元素, 其中 $\mathrm{Ti}$ 来自作为基体材料的纯钛片。复合涂层在烧结前存 在 $\mathrm{C} 、 \mathrm{~N} 2$ 种元素, 说明壳聚糖在电泳沉积过程中共沉积于涂层表面, 而烧结后未测得 $\mathrm{C} 、 \mathrm{~N}$ 元素, 说明烧 结后壳聚糖已完全分解 ${ }^{[10]}$ 。 $\mathrm{Fe}$ 元素的存在, 说明电泳沉积过程中 $\mathrm{Fe}_{2} \mathrm{O}_{3}$ 也同时沉积至钛基体表面,形成 了 $\mathrm{HA} / \mathrm{CS} / \mathrm{Fe}_{2} \mathrm{O}_{3}$ 复合涂层。这从复合涂层明显的浅红褐色也可得到说明。由表 1 可知, $\mathrm{Fe}$ 元素所占的 质量分数 $<1.00 \%$, 处于微量范畴。

表 1 烧结前后复合涂层表面各元素的百分含量

Table 1 Percentages of elements in the surface of composite coating before and after sintering

\begin{tabular}{|c|c|c|c|c|}
\hline \multirow{2}{*}{ Elements } & \multicolumn{2}{|c|}{ Before sintering } & \multicolumn{2}{|c|}{ After sintering } \\
\hline & $w($ Ele $) / \%$ & $x($ Ele $) / \%$ & $w($ Ele $) / \%$ & $x($ Ele $) / \%$ \\
\hline $\mathrm{O}$ & 46.12 & 63.43 & 55.89 & 74.70 \\
\hline $\mathrm{P}$ & 14.84 & 10.50 & 14.79 & 10.21 \\
\hline $\mathrm{Ca}$ & 23.53 & 12.89 & 23.72 & 12.66 \\
\hline $\mathrm{Ti}$ & 10.56 & 4.84 & 4.62 & 2.06 \\
\hline $\mathrm{C}$ & 3.85 & 7.03 & - & - \\
\hline $\mathrm{N}$ & 0.75 & 1.17 & - & - \\
\hline $\mathrm{Fe}$ & 0.35 & 0.14 & 0.98 & 0.37 \\
\hline Total & 100.00 & 100.00 & 100.00 & 100.00 \\
\hline
\end{tabular}

\section{3 烧结处理后复合涂层的 SEM 表面形貌}

经 $700{ }^{\circ} \mathrm{C}$ 热处理 $2 \mathrm{~h}$ 后电泳沉积制得的 $\mathrm{HA} / \mathrm{CS} / \mathrm{Fe}_{2} \mathrm{O}_{3}$ 复合涂层与 $\mathrm{HA}$ 涂层的表面 $\mathrm{SEM}$ 照片如图 3 所示。从图 3 可以看出, HA/CS 涂层经 $700{ }^{\circ} \mathrm{C}$ 烧结处理后, 涂层表面均匀致密。而当 $\mathrm{HA} / \mathrm{CS} / \mathrm{Fe}_{2} \mathrm{O}_{3}$ 悬 浮液质量比为 100:100: 1 时所制得的复合涂层, 经烧结处理后获得的 $\mathrm{HA} / \mathrm{Fe}_{2} \mathrm{O}_{3}$ 复合涂层表面粗粘且孔 洞分布均匀 (图 $3 B$ ), 这些孔洞的形成是经烧结后 CS 热分解或燃烧产生气体所致 ${ }^{[10]}$ 。但由于 HA 在烧 结处理过程中存在收缩致密的特性,因此观察到的复合涂层表面孔洞的孔径远小于 CS 颗粒粒径。
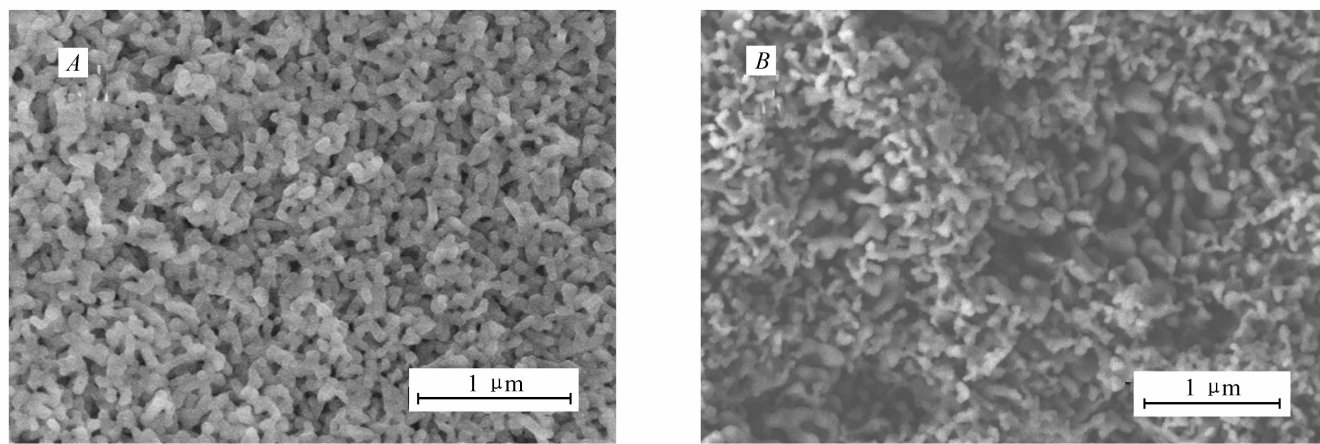

图 3 经 $700{ }^{\circ} \mathrm{C}$ 烧结 $2 \mathrm{~h}$ 后得到的 $\mathrm{HA}$ 涂层 $(A)$ 与 $\mathrm{HA} / \mathrm{Fe}_{2} \mathrm{O}_{3}$ 复合涂层 $(B)$ 的 $\mathrm{SEM}$ 照片

Fig. 3 SEM images of $\mathrm{HA}$ coating $(A)$ and $\mathrm{HA} / \mathrm{Fe}_{2} \mathrm{O}_{3}$ composite coating $(B)$ obtained after sintering at $700{ }^{\circ} \mathrm{C}$ for $2 \mathrm{~h}$

\section{4 复合涂层在 SBF 中的耐腐蚀性能}

图 4 为 $\mathrm{HA}$ 涂层和 $\mathrm{HA} / \mathrm{Fe}_{2} \mathrm{O}_{3}$ 复合涂层在 $1.5 \mathrm{SBF}$ 中的 Nyquist 谱图。从图 4 可看出,在测试频率范 围 $\left(10^{-2} \sim 10^{5} \mathrm{~Hz}\right)$ 内, $\mathrm{HA}$ 复合涂层和 $\mathrm{HA} / \mathrm{Fe}_{2} \mathrm{O}_{3}$ 复合涂层在 $\mathrm{SBF}$ 中均可采用 $R_{1}\left(R_{2} C_{1}\right)\left[\left(R_{3} W\right) C_{2}\right]$ 等 效电路模拟, 其 $R_{2}$ 的阻值分别为 1150 和 $1387 \Omega$, 说明掺入微量 $\mathrm{Fe}$ 元素的复合涂层的抗腐蚀性得到一 定程度的提高, 这可能是由于 $\mathrm{Fe}_{2} \mathrm{O}_{3}$ 是非导体, 掺人到复合涂层中提高了复合涂层禁带电子能级, 从而 使复合涂层的阻值增大, 耐腐蚀性增强。 

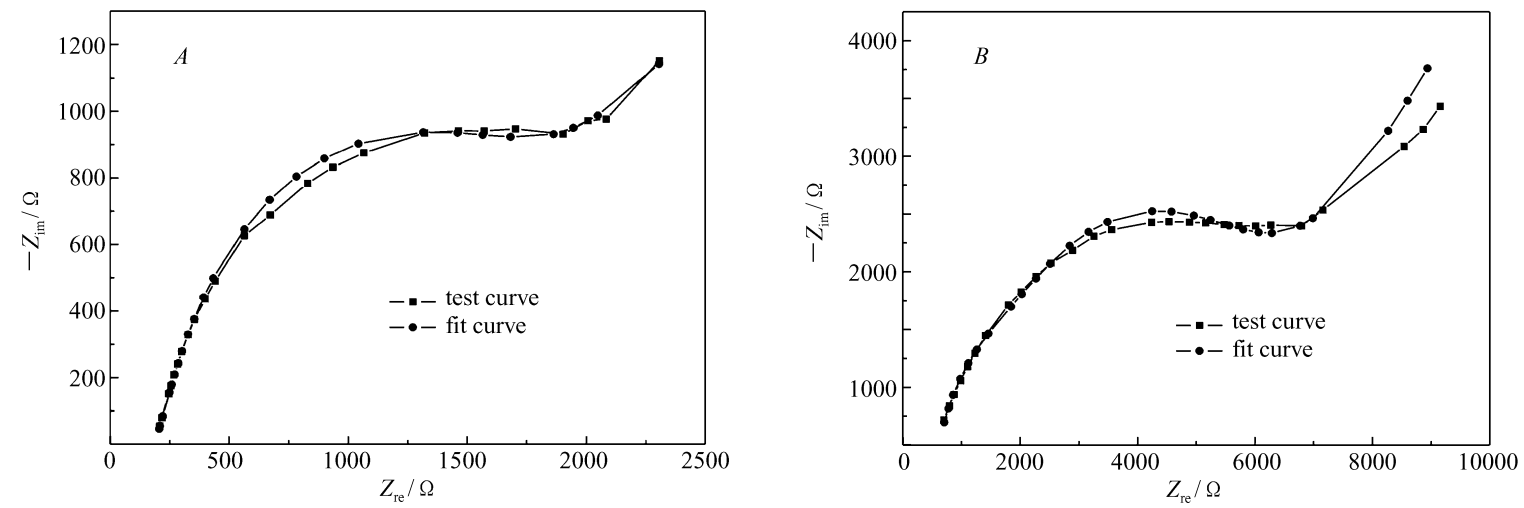

图 $4 \mathrm{HA}$ 涂层 $(A)$ 和 $\mathrm{HA} / \mathrm{Fe}_{2} \mathrm{O}_{3}$ 复合涂层 $(B)$ 在 $1.5 \mathrm{SBF}$ 中的 Nyquist 谱图

Fig. 4 The Nyquist plots of $\mathrm{HA}$ coating $(A)$ and $\mathrm{HA} / \mathrm{Fe}_{2} \mathrm{O}_{3}$ composite coating $(B)$ in $1.5 \mathrm{SBF}$

\section{$2.5 \mathrm{HA} / \mathrm{Fe}_{2} \mathrm{O}_{3}$ 复合涂层的结合强度}

表 2 列出了添加不同质量分数 $\mathrm{Fe}_{2} \mathrm{O}_{3}$ 经 $700{ }^{\circ} \mathrm{C}$ 烧结 $2 \mathrm{~h}$ 后制得的复合涂层与基体钛片的结合强度。 从表 2 可以看出, $\mathrm{HA} / \mathrm{CS} / \mathrm{Fe}_{2} \mathrm{O}_{3}$ 质量比为 50:50:1 的复合涂层的结合强度最大, 达到 $28.5 \mathrm{MPa}$ 。 $\mathrm{HA} / \mathrm{Fe}_{2} \mathrm{O}_{3}$ 复合涂层结合强度高于纯 $\mathrm{HA}$ 涂层的原因, 可能是由于 $\mathrm{HA}$ 涂层烧结时的体积收缩及 $\mathrm{HA}$ 与 基体钛热膨胀系数 $\left(\alpha_{\mathrm{Ti}}=8.7 \times 10^{-6} \mathrm{~K}^{-1} ; \alpha_{\mathrm{HA}}=13.6 \times 10^{-6} \mathrm{~K}^{-1}\right)$ 的不匹配, 导致热处理过程中涂层与基 体界面处积累较大的残余应力,而加人 $\mathrm{Fe}_{2} \mathrm{O}_{3}$ 制得的复合涂层中虽然铁元素的含量小于 $1 \%$,但可使复 合涂层中游离氧的含量降低, 同时使磷酸盐涂层原有的层状结构向架状结构转变, 从而使 HA 涂层的热 膨胀系数降低,趋向于与钛的热膨胀系数匹配 ${ }^{[14]}$ 。

表 2 添加不同质量分数 $\mathrm{Fe}_{2} \mathrm{O}_{3}$ 对复合涂层与基体结合强度的影响

Table 2 Effect of $\mathrm{Fe}_{2} \mathrm{O}_{3}$ mass ratio on adhesion between the composite coating and substrate

\begin{tabular}{cc||cc}
\hline$m(\mathrm{HA}): m(\mathrm{CS}): m\left(\mathrm{Fe}_{2} \mathrm{O}_{3}\right)$ & Bonding strength $\sigma / \mathrm{MPa}$ & $m(\mathrm{HA}): m(\mathrm{CS}): m\left(\mathrm{Fe}_{2} \mathrm{O}_{3}\right)$ & Bonding strength $\sigma / \mathrm{MPa}$ \\
\hline $1: 1: 0$ & 19.5 & $50: 50: 1$ & 28.5 \\
$10: 10: 1$ & 22.1 & $100: 100: 1$ & 27.5 \\
\hline
\end{tabular}

\section{$2.6 \mathrm{HA} / \mathrm{Fe}_{2} \mathrm{O}_{3}$ 复合涂层生物活性评价}

图 5 为采用 $\mathrm{HA} / \mathrm{CS} / \mathrm{Fe}_{2} \mathrm{O}_{3}$ 质量比为 100:100:1 制得的 $\mathrm{HA} / \mathrm{Fe}_{2} \mathrm{O}_{3}$ 复合涂层于 1.5SBF 中浸泡 7 和 $14 \mathrm{~d}$ 后的 SEM 照片。从图 5 可以看出, 浸泡 $7 \mathrm{~d}$ 后 $(A) \mathrm{HA} / \mathrm{Fe}_{2} \mathrm{O}_{3}$ 复合涂层表面形成球状颗粒, 呈簇状重 叠生长, 有利于碳磷灰石 $(\mathrm{CHA})$ 层的形成 ${ }^{[15]}$ 。浸泡 $14 \mathrm{~d}$ 后 $(B)$ 复合涂层表面呈现均匀的花瓣状的片状 结构,完全被新生成的 CHA 层所覆盖。从以上 SEM 照片可以看出, 差基磷灰石在模拟体液中并非整体 溶解, 而是在逐渐溶解的同时诱导 CHA 层沉积, 是 1 个分区域分层次进行的过程 ${ }^{[16]}$ 。
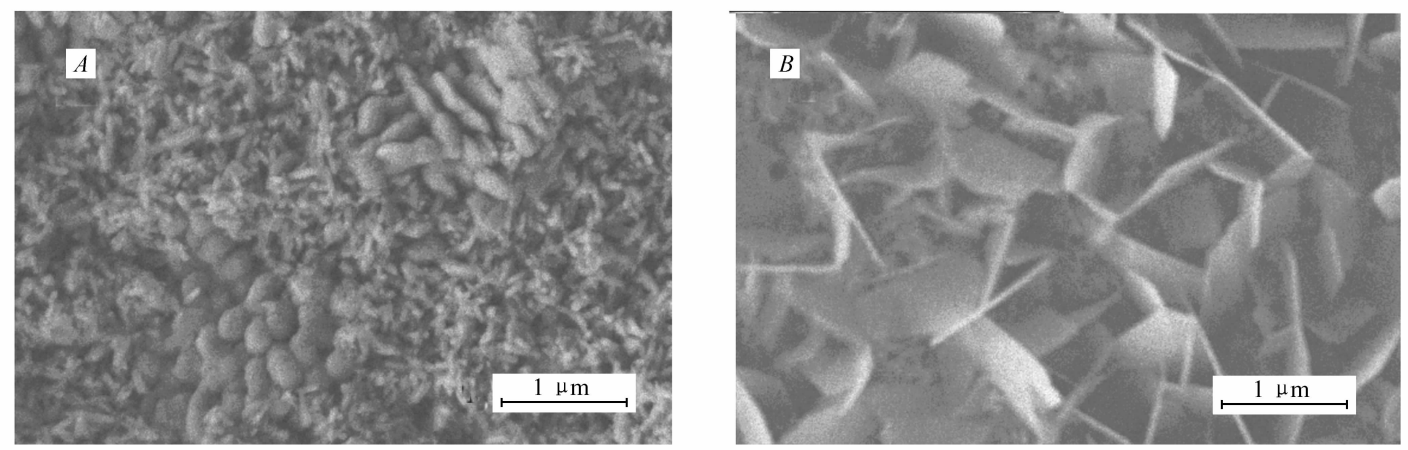

图 $5 \mathrm{HA} / \mathrm{Fe}_{2} \mathrm{O}_{3}$ 复合涂层在 $1.5 \mathrm{SBF}$ 中浸泡 $7 \mathrm{~d}(A)$ 和 $14 \mathrm{~d}(B)$ 的 SEM 照片

Fig. 5 Surface morphologies of $\mathrm{HA} / \mathrm{Fe}_{2} \mathrm{O}_{3}$ composite coating after immersion in $1.5 \mathrm{SBF}$ for $7 \mathrm{~d}(A)$ and $14 \mathrm{~d}(B)$ 
图 6 为烧结后复合涂层以及在 $1.5 \mathrm{SBF}$ 中分别 培养 7 和 $14 \mathrm{~d}$ 的 FT-IR 谱图。从图 6 可以看出, $984 \mathrm{~cm}^{-1}$ 对应于 $\mathrm{PO}_{4}^{3-}$ 的 $\nu_{1}$ 振动吸收峰, 随着培养天 数的增加而逐渐减弱, 1094、1044 和 $984 \mathrm{~cm}^{-1}$ 处的 吸收峰出现合并同时峰形变宽,与此同时 $\mathrm{OH}^{-}$特征 峰也随着培养天数的增加而弱化。由此可以推测, 体系中的 $\mathrm{PO}_{4}^{3-}$ 所处的结构环境发生较大程度的变 化,一般认为,其可能的原因是在 SBF 浸泡过程中, $\mathrm{PO}_{4}^{3-}$ 和 $\mathrm{OH}^{-}$被 $\mathrm{CO}_{3}^{2-}$ 部分取代 ${ }^{[17]}$, 但由于测试该红 外谱图时, 直接将 $\mathrm{CO}_{2}$ 的吸收峰作为背景值扣除, 因 此在该谱图中无明显的 $\mathrm{CO}_{3}^{2-}$ 吸收峰。

表 3 中列出了经烧结处理后在 1.5SBF 中培养 $14 \mathrm{~d}$ 的 $\mathrm{HA} / \mathrm{Fe}_{2} \mathrm{O}_{3}$ 复合涂层的 EDS 微区元素分析数 据。与表 1 相比新增了碳元素。从 CHA 的形成机

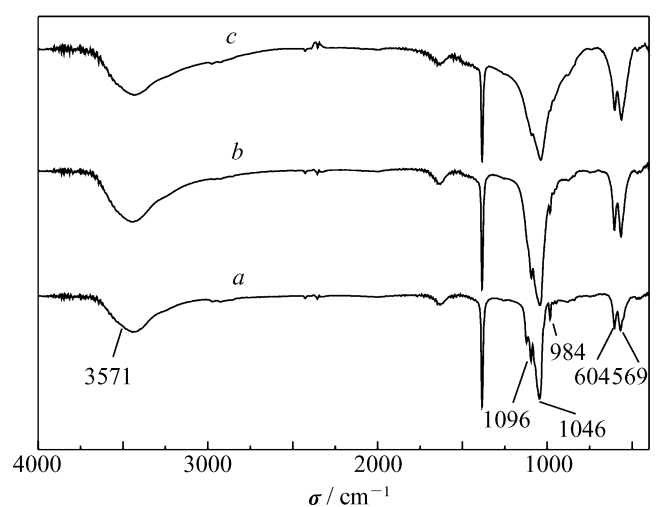

图 6 不同阶段处理所得的复合涂层的 FT-IR 谱图 Fig. 6 FT-IR spectra of composite coatings obtained from different processing $a$. after sintering; $b$. after immersion in $1.5 \mathrm{SBF}$ for 7 days; c. after immersion in $1.5 \mathrm{SBF}$ for 14 days 制可以说明浸泡后覆盖在 $\mathrm{HA} / \mathrm{Fe}_{2} \mathrm{O}_{3}$ 复合涂层表面 上有新物质 $\mathrm{CHA}$ 生成, 因为 $\mathrm{HA} / \mathrm{Fe}_{2} \mathrm{O}_{3}$ 复合涂层容易诱导 $\mathrm{Ca}^{2+} 、 \mathrm{PO}_{4}^{3-}$ 和 $\mathrm{OH}^{-}$不断与 $\mathrm{SBF}$ 发生离子交换 形成 CHA, 而 CHA 能选择性吸附血清中某些功能蛋白 ${ }^{[18]}$, 促使骨细胞增殖、分化, 从而使新生骨组织直 接在复合涂层表面形成 ${ }^{[19]}$ 。由于 $\mathrm{EDS}$ 测试的是涂层表面各元素组成, 因此在 $\mathrm{HA} / \mathrm{Fe}_{2} \mathrm{O}_{3}$ 复合涂层表面 形成 CHA 层覆盖后, 涂层中铁元素的含量明显降低。涂层表面的碳磷灰石化的程度常被用于评价涂层 的生物活性 ${ }^{[20]}$,据此推测该 $\mathrm{HA} / \mathrm{Fe}_{2} \mathrm{O}_{3}$ 复合涂层具有良好的诱导骨生长的生物活性。

\section{表 3 经 $1.5 \mathrm{SBF}$ 培养 $14 \mathrm{~d}$ 后 $\mathrm{HA} / \mathrm{Fe}_{2} \mathrm{O}_{3}$ 复合涂层表面各元素的百分含量}

Table 3 Percentage of elements in the surface of $\mathrm{HA} / \mathrm{Fe}_{2} \mathrm{O}_{3}$ composite coating after immersion in 1.5SBF for 14 days

\begin{tabular}{ccc}
\hline Elements & $w($ Ele $) / \%$ & $x($ Ele $) / \%$ \\
\hline O & 45.24 & 59.90 \\
$\mathrm{P}$ & 18.09 & 12.37 \\
$\mathrm{Ca}$ & 29.71 & 15.70 \\
$\mathrm{C}$ & 6.78 & 11.96 \\
$\mathrm{Fe}$ & 0.18 & 0.07 \\
Total & 100.00 & 100.00 \\
\hline
\end{tabular}

\section{3 结 论}

$\mathrm{HA} / \mathrm{CS} / \mathrm{Fe}_{2} \mathrm{O}_{3}$ 质量比为 100: 100: 1 的悬浮液电泳沉积制备的 $\mathrm{HA} / \mathrm{CS} / \mathrm{Fe}_{2} \mathrm{O}_{3}$ 复合涂层, 经 $700{ }^{\circ} \mathrm{C}$ 烧 结 $2 \mathrm{~h}$ 制得的 $\mathrm{HA} / \mathrm{Fe}_{2} \mathrm{O}_{3}$ 复合涂层表面粗粘, 与基体结合强度 $(27.5 \mathrm{MPa}$ )较纯 $\mathrm{HA}$ 涂层有很大程度的提 高。电化学测试结果表明, 与 $\mathrm{HA}$ 涂层相比, $\mathrm{HA} / \mathrm{Fe}_{2} \mathrm{O}_{3}$ 复合涂层在 $1.5 \mathrm{SBF}$ 中耐腐蚀性有显著增强, 作为 成骨替代材料植人人体后可延长使用周期。生物活性评价结果表明,添加 $\mathrm{Fe}_{2} \mathrm{O}_{3}$ 后复合涂层呈现良好的 诱导骨生长的生物活性, 因此该 $\mathrm{HA} / \mathrm{Fe}_{2} \mathrm{O}_{3}$ 复合涂层有望通过相关的生物相容性和安全性研究后, 开发 成为新一代骨替代植人生物陶瓷材料。

\section{参 考 文 献}

[1] WANG Wenjing, HUANG Jianfeng, LI Yinghua, et al. Theoretical Research and Progress of Basal Body and Bio-coatings in Biomaterial Filed $[\mathrm{J}]$. J Clin Rehab Tissue Eng Res,2010,14(3):30-33(in Chinese).

王文静, 黄剑锋, 李颖华, 等. 生物材料中基体及涂层材料的研究与进展 $[\mathrm{J}]$. 中国组织工程研究与临床康复, 2010, $14(3): 30-33$. 
[2] He X,Huang X,Lu Q, et al. Nanoscale Control of Silks for Regular Hydroxyapatite Formation[J]. Prog Nat Sci:Mater Int, $2012, \mathbf{2 2}(2): 115-119$.

[3] Li D, Wang W, Guo R, et al. Restoration of Rat Calvarial Defects by Poly (lactide-co-glycolide)/hydroxyapatite Scaffolds Loaded with Bone Mesenchymal Stem Cells and DNA Complexes[J]. Chinese Sci Bull,2012,57(5):435-444.

[4] SUN Baoguo, CHEN Fengbao, ZHAI Fuying, et al. Determination of the Contents of Traceelements in Human Bones [J]. Henan Med Res,1997,6(2):100-101 (in Chinese).

孙保国,陈凤苞,翟福英, 等. 人体骨骼中微量元素的测定 $[J]$. 河南医学研究,1997,6(2):100-101.

[5] Wu T , Nan K, Chen J,et al. A New Bone Repair Scaffold Combined with Chitosan/hydroxyapatite and Sustained Releasing Icariin [J]. Chinese Sci Bull,2009,54:2953-2961.

[6] YU Rong, FENG Bo, WANG Jianxin, et al. Protein-hydroxyapatite Composite Coatings on $\mathrm{TiO}_{2}$ Nanotube Layers and Bond Strength to Substrate[J]. Chinese J Inorg Mater,2011,26(9):987-992(in Chinese).

于榕, 冯波, 汪建新, 等. $\mathrm{TiO}_{2}$ 纳米管表面蛋白质-羟基磷灰石复合涂层及其结合强度 $[\mathrm{J}]$. 无机材料学报, 2011, 26(9):987-992.

[7] LI Zhihong, WU Jimin, HUANG Shujie, et al. Preparation and Property of Strontium-substituted Hydroxyapatite [J]. Chinese J Inorg Mater,2011,26(1):49-54 (in Chinese).

李志宏,武继民,黄姝杰, 等. 掺锶羟基磷灰石的制备与性能研究 [J]. 无机材料学报,2011,26(1):49-54.

[8] Wei Z J, Wang C Y, Zou S W, et al. $\mathrm{Fe}_{2} \mathrm{O}_{3}$ Nanoparticles as Particulate Emulsifier: Preparation of Magnetic and Biocompatible PLGA Microcapsules[J]. Colloids Surf A :Physicochem Eng Aspects, 2011,392 (1):116-123.

[9] Li G D, Feng S Y,Zhou D L. Magnetic Bioactive Glass Ceramic in the System $\mathrm{CaO}-\mathrm{SiO}_{2}-\mathrm{P}_{2} \mathrm{O}_{5}-\mathrm{MgO}_{2}-\mathrm{Fe}_{2} \mathrm{O}_{3}$ for $\mathrm{Hyperthermia}$ Treatment of Bone Tumor[J]. J Mater Sci:Mater Med,2011,22 (10) :2197-2206.

[10 ] LIU Rongfang, XIAO Xiufeng, NI Jun, et al. Study on Kinetics of Hydrothermal Synthesis of Hydroxyapatite Powders [J]. Chinese J Inorg Chem,2003,19(10):1079-1084( in Chinese).

刘榕芳, 肖秀峰, 倪军, 等. 差基磷灰石粉末的水热合成及动力学研究 $[\mathrm{J}]$. 无机化学学报, 2003,19(10): 10791084.

[11] ZHANG Lan,HUANG Ziyang. Fabrication and Bioactivity of Porous Hydroxyapatite Coating by Electrophoretic Deposition Using Different Porogens[J]. Chinese J Appl Chem,2012,29(1):46-50(in Chinese).

张岗,黄紫洋. 造孔剂对电沉积制备多孔 HA 涂层及其生物活性的影响 [J]. 应用化学, 2012,29(1):46-50.

[12] HUANG Ziyang, ZHANG Lan, LIANG Guangchao. Study on Fabrication and Properities of Porous $\mathrm{HA}_{2} \mathrm{SiO}_{2} \mathrm{Composite}$ Coating on Tiatanium Substrate[J]. Acta Chim Sin,2012,70(3):235-240( in Chinese).

黄紫洋,张岗,梁广超. 钛基多孔 $\mathrm{HA} / \mathrm{SiO}_{2}$ 复合涂层的制备及其性能研究 $[\mathrm{J}]$. 化学学报,2012,70(3):235-240.

[13] XIAO Xiufeng, LIU Rongfang, ZHANG Xiaoqin, et al. Study on Hydrothermal Synthesis of Nanosized Hydroxyapatite Powders [J]. Inorg Chem Ind,2004,36(3):16-18( in Chinese).

肖秀峰,刘榕芳,张晓勤, 等. 水热合成纳米羟基磷灰石粉体的研究 $[\mathrm{J}]$. 无机盐工业,2004,36(3):16-18.

[14] LI Shengchun, CHEN Pei, LI Yaogang. Influence of $\mathrm{Fe}_{2} \mathrm{O}_{3}$-dop on Thermal Property of $\mathrm{ZnO}_{2}-\mathrm{B}_{2} \mathrm{O}_{3}-\mathrm{P}_{2} \mathrm{O}_{5}-\mathrm{R}_{n} \mathrm{O}_{m}$ Glass System [J]. New Chem Mater ,2011,39(7):114-116,133 (in Chinese).

李胜春, 陈培, 李耀刚. $\mathrm{Fe}_{2} \mathrm{O}_{3}$ 掺杂对 $\mathrm{ZnO}-\mathrm{B}_{2} \mathrm{O}_{3}-\mathrm{P}_{2} \mathrm{O}_{5}-\mathrm{R}_{n} \mathrm{O}_{m}$ 玻璃热力学性能的影响 $[\mathrm{J}]$. 化工新型材料, 2011, 39(7) : 114-116,133.

[15] HUANG Yong, CAO Yu, HE Congjun, et al. The Present Situation of Hydroxyapatite/ $\mathrm{ZrO}_{2}$ Bioactive Composite Coating [J]. Life Sci Instrum, 2010,8:30-33(in Chinese).

黄勇, 曹郁, 和从军, 等. 羟基磷灰石/氧化锆生物活性复合涂层的研究 $[\mathrm{J}]$. 生命科学仪器, 2010,8:30-33.

[16] LI Zhouxin, WU Yongzhi, HE Dingyong, et al. Micro-plasma Sprayed Zirconia Reinforced Hydroxyapatite Composite Coatings [J]. Trans China Weld Inst,2008,29(6):1-4(in Chinese).

栗卓新, 吴永智, 贺定勇, 等. 微束等离子喷涂氧化锆增韧羟基磷灰石复合涂层 $[\mathrm{J}]$. 焊接学报,2008,29(6):1-4.

[17] Ni S Y, Chang J, Chou L. A Novel Bioactive Porous $\mathrm{CaSiO}_{3}$ Scaffold for Bone Tissue Engineering[J]. Biomed Mater Res A, $2006,76(1): 196-205$.

[18] Na Y, Chen S, Ouyang Y, et al. Bomimetic Mineralization Synthesis of Hydroxyapatite Bacterial Cellulose Nanocomposites [J]. Prog Nat Sci:Mater Int ,2011,21:472-477.

[19] Li P, Kanagasnie I, Degroot K, et al. Bone Like Hydroxyapatite Induction by a Gel-derived Titania on a Titanium Substrate [J]. J Am Ceram Soc,1994,77 (5):1307-1312.

[20] Kokubo T,Kim H M, Kawashita M, et al. Bioactive Metals:Preparation and Properties[J]. J Mater Sci:Mater Med,2004, $\mathbf{1 5}(2): 99-107$. 


\title{
Preparation and Bioactivity for Inducing Bone Growth of Hydroxyapatite $/ \mathrm{Fe}_{2} \mathrm{O}_{3}$ Composite Coating
}

\author{
LI Longzhu, HUANG Ziyang * , LIN Yan, CAO Mei, LIN Jianqun \\ (College of Chemistry and Chemical Engineering, Fujian Normal University, Fuzhou 350007, China)
}

Abstract Electrophoretic deposition ( EPD) has been exploited for the preparation of hydroxyapatite/ chitosan/ferric oxide $\left(\mathrm{HA} / \mathrm{CS} / \mathrm{Fe}_{2} \mathrm{O}_{3}\right)$ composite coating, which was then sintered at $700{ }^{\circ} \mathrm{C}$ for $2 \mathrm{~h}$ to yield $\mathrm{HA} / \mathrm{Fe}_{2} \mathrm{O}_{3}$ composite coating. The surface morphology, phase composition, corrosion resistance and adhesion between the composite coating and substrate were characterized by SEM, EDS and XRD, FT-IR, electrochemical instrument and Lloyd LR5K Plus Testing Instrument, respectively. The bioactivity for inducing bone growth of the $\mathrm{HA} / \mathrm{Fe}_{2} \mathrm{O}_{3}$ composite coating was evaluated in 1.5 times of ionic concentration of simulated body fluid(1.5SBF). When the mass ratio of $\mathrm{HA}: \mathrm{CS}: \mathrm{Fe}_{2} \mathrm{O}_{3}$ in the suspension was 100:100:1, the resultant $\mathrm{HA} / \mathrm{Fe}_{2} \mathrm{O}_{3}$ composite coating had rough surface and strong adhesion up to 27. $5 \mathrm{MPa}$ with substrate and showed high corrosion resistance in $1.5 \mathrm{SBF}$ and excellent bioactivity for inducing bone growth in $1.5 \mathrm{SBF}$.

Keywords hydroxyapatite $/ \mathrm{Fe}_{2} \mathrm{O}_{3}$ composite coating, electrophoretic deposition, bioactivity

\section{《应用化学》2014 年征订启事}

《应用化学》创刊于 1983 年, 是经国家科委批准向国内、国外公开发行的学术性期刊。由中国科学院主管, 中国化 学会和中国科学院长春应用化学研究所主办,科学出版社出版。为中国科技核心期刊。

《应用化学》设有综合评述、研究论文、研究简报、研究快报栏目。出版周期短,报道新成果快。

《应用化学》期刊被 14 家国内外重要检索机构、文摘收录。

《应用化学》面向科研单位、大专院校和化学化工领域的科研技术人员。

本刊承揽各类化学、化工材料、分析测试仪器及各类化学产品介绍和相关领域科技信息等广告业务。

《应用化学》投稿全部采用网上投稿方式 (http://yyhx. ciac. jl. cn 点击“网上投稿”或“投稿注册”, 按照提示步骤操 作)。

-中国科学院主管,中国化学会和中国科学院长春应用化学研究所主办。

- 多次获国家、省、部级奖励, 发行量大,广告宣传效果好。

- 国内外公开发行, 月刊, 每月 10 日出版。

- 国内统一刊号 CN 22-1128/06; 国际标准刊号 ISSN 1000-0518。

- 全国各地邮局订阅, 国内邮发代号 8-184; 每册定价 30.00 元, 全年定价 360 元

-广告经营许可证号:吉工商广字 206 号

- 中国国际图书贸易总公司办理国外订阅( 国外发行代号 BM809)

- 如未能在邮局订阅, 可与编辑部联系订阅。

《应用化学》编辑部地址: 吉林省长春市人民大街 5625 号 邮编: 130022

电话:0431-85262016,85262330

传真:0431-85685653

E-mail: yyhx@ ciac. ac.cn

网 址: http://yyhx. ciac. jl. cn 